\title{
A. HARAUX \\ Développement asymptotique d'intégrales oscillantes lentement convergentes à l'infini
}

Revue française d'automatique, informatique, recherche opérationnelle. Analyse numérique, tome 10, no 2 (1976), p. 49-59.

$<$ http://www.numdam.org/item?id=M2AN_1976_10_2_49_0>

(C) AFCET, 1976, tous droits réservés.

L'accès aux archives de la revue "Revue française d'automatique, informatique, recherche opérationnelle. Analyse numérique » implique l'accord avec les conditions générales d'utilisation (http://www.numdam.org/legal. php). Toute utilisation commerciale ou impression systématique est constitutive d'une infraction pénale. Toute copie ou impression de ce fichier doit contenir la présente mention de copyright.

\section{Numdam}

Article numérisé dans le cadre du programme

Numérisation de documents anciens mathématiques

http://www.numdam.org/ 


\title{
DÉVELOPPEMENT ASYMPTOTIQUE D'INTÉGRALES OSCILLANTES LENTEMENT CONVERGENTES A L'INFINI
}

\author{
par A. Haraux $\left({ }^{1}\right)$
}

Communiqué par P. G. Ciarlet

\begin{abstract}
Résumé. - En vue d'applications à la Physique, on cherche à estimer l'intégrale $\int_{x}^{+\infty} f(t) g(t) d t$ pour de grandes valeurs de $x$, la fonction $f$ étant quasi périodique et nulle en moyenne à l'infini, la fonction g étant à signe très lentement variable et à module tendant lentement vers zéro à l'infini.
\end{abstract}

(i) Si $g$ est convexe, et si $f$ a des primitives bornées d'ordre supérieur à 2 , on obtient un développement asymptotique de l'intégrale.

(ii) Si $g$ est le produit d'une fonction absolument monotone de classe $C^{\infty}$ par un facteur très lentement oscillant à l'infini, et si f est périodique, on obtient une approximation dont la précision varie comme une exponentielle décroissante de $x$.

\section{INTRODUCTION}

Le but de cette étude est d'obtenir une estimation assez précise d'intégrales généralisées de fonctions oscillantes, en vue d'applications à la physique.

En termes vagues, si $f$ est une fonction quasi périodique continue oscillant à l'infini autour de 0 , et $g$ une fonction convexe non sommable tendant vers 0 à l'infini (" facteur d'amortissement »), l'intégrale $\int_{0}^{+\infty} f(t) g(t) d t$ est au plus semi-convergente.

Sa convergence est liée à l'existence pour $f$ d'une primitive bornée, et dans ce cas le « reste " $\int_{x}^{+\infty} f(t) g(t) d t$ tend très lentement vers 0 , ce qui rend fastidieux le calcul numérique de $\int_{0}^{+\infty} f(t) g(t) d t$ par les méthodes classiques.

On va voir que sous des hypothèses raisonnables sur $g$, l'existence pour $f$ de primitives bornées d'ordre supérieur permet un développement asympto-

(1) Analyse Numérique, Tour 55-65, Université Pierre-et-Marie-Curie, Paris VI, Paris.

Revue Française d'Automatique, Informatique et Recherche Opérationnelle, août 1976. 
tique de ce reste, bien préférable à une simple majoration. Dans le cas où $f$ est exactement périodique, et $g$ une fonction $C^{\infty}$, produit d'une fonction absolument monotone par un facteur lentement oscillant à l'infini, c'est-à-dire du type $t^{-\beta} e^{i t^{\alpha}}, 0 \leqq \alpha<1, \beta>0$, on obtiendra un développement très précis, avec décroissance exponentielle du reste à l'infini. Ce calcul contient le cas modèle de l'intégrale

$$
\int_{x}^{+\infty} e^{i k t} d t, \quad k \in R^{*}, \quad \alpha>0
$$

Le problème qui est à l'origine de notre étude m'a été posé par M. Séchaud, chercheur au laboratoire O.P.M. de l'École Polytechnique. Les majorations obtenues ont déjà été utilisées sur ordinateur pour le calcul effectif d'intégrales doubles faisant intervenir l'expression de l'exemple 3 (II).

\section{FONCTIONS PÉRIODIQUES CENTRÉES}

DÉfrnition : Une fonction $f: R \rightarrow C$, périodique de période $T$, localement intégrable, sera dite centrée si

$$
\int_{0}^{T} f(t) d t=0
$$

Dans la suite, $f$ désigne une fonction $T$-périodique.

REMARQUES :

$1^{\circ}$ S'il existe $a \in R$ tel que $f_{a}(t)=f(t+a)$ soit impaire, $f$ est centrée.

$2^{\circ}$ Si $f$ est une fonction $T$-périodique, alors la fonction

$$
x \rightarrow f(x)-\frac{1}{T} \int_{0}^{T} f(t) d t
$$

est $T$-périodique centrée.

$3^{\circ}$ Supposons la fonction $f$ continue. Alors elle est centrée si et seulement si elle admet une primitive $T$-périodique, ou encore si et seulement si elle a une primitive bornée.

$4^{\circ}$ Soit $f$ une fonction continue, 2 П-périodique. Alors elle est centrée si et seulement si la décomposition de $f$ en série de Fourier n'a pas de terme constant.

Proposition $1:$ Sif $: R \rightarrow C$ est une fonction périodique centrée de période $T$, elle possède une primitive centrée et une seule : notons-la $f_{(1)}$. On peut alors construire par itération $f_{(2)}, f_{(3)}, \ldots, f_{(n)}, \ldots$

(i) On $a\left\|f_{(1)}\right\|_{\infty} \leqq T / 4\|f\|_{\infty}$. 
(ii) D'autre part, si $T=2 \Pi$, on a $\left\|f_{(1)}\right\|_{L^{2}} \leqq\|f\|_{L^{2}}$, et en particulier la suite $\left(f_{(n)}\right.$ est uniformément bornée (puisque $\|f\|_{\infty} \leqq C\left(\|f\|_{L^{2}}+\left\|f^{\prime}\right\|_{L^{2}}\right.$ ).

Démonstration: D'après la théorie de la transformation de Fourier,

$$
f(x)=\sum_{\text {p.p. } k \in Z^{*}} C_{k} e^{i k x}, \quad \text { avec } \quad \sum_{k \in Z^{*}}\left|C_{k}\right|^{2}=\frac{1}{2 \Pi} \int_{-\Pi}^{+\Pi}|f(x)|^{2} d x<+\infty .
$$

Il vient facilement

$$
f_{(n)}(x)=\sum_{k \in Z^{*}} \frac{C_{k}}{(i k)^{n}} e^{i k x}, \quad \forall n \geqq 1,
$$

d'où l'on déduit

$$
\left\|f_{(1)}\right\|_{L^{2}}^{2}=2 \Pi \sum_{k \in Z^{*}} \frac{\left|C_{k}^{2}\right|}{k^{2}} \leqq 2 \Pi \sum_{k \in Z^{*}}\left|C_{k}\right|^{2}=\|f\|_{L^{2}}^{2},
$$

ce qui démontre (ii).

On peut obtenir directement un majorant uniforme de $\left|f_{(n)}\right|_{\infty}^{\prime}$ en remarquant que, pour $n \geqq 1$,

$$
\left\|f_{(n)}\right\|_{\infty} \leqq \sum_{k \in Z^{*}} \frac{\left|C_{k}\right|}{|k|^{n}} \leqq \sum_{k \in Z^{*}} \frac{\left|C_{k}\right|}{|k|}<+\infty .
$$

L'application $f \rightarrow f_{(1)}$ est linéaire. Quitte à remplacer $f$ par $R_{e}(u . f)$, où $u$ est un nombre complexe de module arbitraire, on peut supposer $f$ à valeurs réelles. Dans ce cas, $f_{(1)}$ est aussi à valeurs réelles et centrées. Soit $x \in R$ un réel quelconque : les deux nombres $\int_{x}^{x+(r / 2)} f_{(1)}(t) d t$ et $\int_{x-(T / 2)}^{x} f_{(1)}(t) d t$ sont opposés donc de signes contraires.

Si par exemple $\int_{x}^{x+(T / 2)} f_{(1)}(t) d t$ est négatif, alors

$$
f_{(1)}(x)-f_{(1)}(t)=\int_{t}^{x} f(\theta) d \theta \leqq|x-t|\|f\|_{\infty}, \quad \forall t \in R,
$$

et donc, intégrant de $x$ à $x+(T / 2)$,

$$
{ }_{2}^{T} f_{(1)}(x)-\int_{x}^{x+(T / 2)} f_{(1)}(t) d t \leqq \frac{T^{2}}{8}\|f\|_{\infty}
$$

et

$$
f_{(1)}(x) \leqq{ }_{4}^{\mathrm{T}}\|f\|_{\infty} .
$$

août 1976. 
Si c'est le cas contraire, on intègre de $(x-(T / 2))$ à $x$, et on obtient le même résultat. Finalement, changeant $f$ en $-f$, on a aussi

$$
-f_{(1)}(x) \leqq \frac{T}{4}\|-f\|_{\infty}=\frac{T}{4}\|f\|_{\infty} .
$$

D'où en définitive

$$
\left\|f_{(1)}\right\|_{\infty} \leqq \frac{T}{4}\|f\|_{\infty}
$$

ce qui démontre (i).

\section{DÉVELOPPEMENTS ASYMPTOTIQUES D'ORDRE FINI}

\section{1. Étude générale}

LEMME FONDAMENTAL : Si $\gamma:[X,+\infty] \rightarrow R^{+}$est une fonction continue décroissante, et $F:\left[X,+\infty\left[\rightarrow C\right.\right.$ une fonction bornée de classe $C^{1}$, on a

$$
\left|\int_{a}^{b} F^{\prime}(t) \gamma(t) d t\right| \leqq 2 \gamma(a) \sup _{[X,+\infty[}|F| \quad \text { pour } \quad X \leqq a \leqq b .
$$

Démonstration : $\alpha$ ) Toute fonction $C^{0}$ décroissante positive est limite uniforme sur tout compact d'une suite de fonctions $C^{1}$ (et même $C^{\infty}$ ) décroissantes positives.

B) Dans le cas où $\gamma$ est de classe $C^{1}$, on a

$$
\begin{aligned}
\left|\int_{a}^{b} F^{\prime}(t) \gamma(t) d t\right| & =\left|[\gamma F]_{a}^{b}-\int_{a}^{b} F(t) \gamma^{\prime}(t) d t\right| \\
& \leqq \sup _{[x,+\infty[}|F|\left\{\gamma(a)+\gamma(b)+\int_{a}^{b}\left|\gamma^{\prime}(t)\right| d t\right\} \\
& =2 \gamma(a) \sup _{[x,+\infty[}|F|,
\end{aligned}
$$

pour $X \leqq a \leqq b$.

ThÉORÈME 1 : Soit $f, F, \Phi$ trois fonctions continues de $[A,+\infty[$ dans $C$ telles que

$$
\begin{aligned}
& |F| \leqq M<+\infty \quad \text { sur }\left[A,+\infty\left[, \quad F^{\prime}=f,\right.\right. \\
& |\Phi| \leqq P<+\infty \quad \text { sur }\left[A,+\infty\left[, \quad \Phi^{\prime}=F,\right.\right.
\end{aligned}
$$

et $g:\left[A,+\infty\left[\rightarrow R\right.\right.$ une fonction convexe de classe $C^{1}$ telle que $\lim g(x)=0$. Alors :

(i) L'intégrale $\int_{A}^{+\infty} f(t) g(t) d t$ est convergente. 
(ii) On $a$

$$
\int_{x}^{+\infty} f(t) g(t) d t=g(x)[-F(x)+\lambda(x)]
$$

$o \dot{u}$

$$
\lambda(x) \leqq 2 P\left|\frac{g^{\prime}(x)}{g(x)}\right| \quad(\text { on prend } g>0)
$$

Démonstration :

(i) résulte trivialement du fait que $f$ a une primitive bornée.

(ii)

$$
\int_{x}^{+\infty} f(t) g(t) d t+g(x) F(x)=-\int_{x}^{+\infty} g^{\prime}(t) F(t) d t
$$

Posant $g^{\prime}=-\gamma$, le lemme fondamental donne

$$
|\lambda(x)| \leqq \frac{1}{g(x)} \times 2 \gamma(x)=2 P\left|\frac{g^{\prime}(x)}{g(x)}\right|
$$

REMARQUe : Dans les applications $\lim _{x \rightarrow+\infty}\left|g^{\prime}(x) / g(x)\right|=0$. On aura même en général $\left|g^{\prime}(x) / g(x)\right| \leqq K / x$ pour $x$ assez grand.

Corollaire $1:$ Soit $g:\left[A,+\infty\left[\rightarrow R\right.\right.$ une fonction de classe $C^{0}$ telle que, pour $p \in\{0,1, \ldots, n\},(-1)^{p} g^{(p)} \geqq 0$, et telle que la fonction $\left|g_{n}\right|$ soit. décroissante. On suupose que

$$
\lim _{x \rightarrow+\infty} g(x)=\lim _{x \rightarrow+\infty}\left|\frac{g^{\prime}(x)}{g(x)}\right|=\ldots=\lim _{x \rightarrow+\infty}\left|\frac{g^{(n)}(x)}{g^{(n-1)}(x)}\right|=0 .
$$

Si alors $f, F_{1}, F_{2}, \ldots, F_{n+1}$ sont des fonctions à valeurs complexes bornées sur $\left[A,+\infty\left[\right.\right.$ telles que $F_{n+1}^{\prime}=F_{n}, F_{n}^{\prime}=F_{n-1}, \ldots, \mathrm{F}_{1}^{\prime}=f$, la fonction étant continue, l'intégrale $\int_{A}^{+\infty} f(t) g(t) d t$ est convergente, et

où

$$
\begin{aligned}
\int_{A}^{+\infty} f(t) g(t) d t= & -g(x) F_{1}(x)+g^{\prime}(x) F_{2}(x)+\ldots \\
& +(-1)^{n} g^{(n-1)}(x)\left[F_{n}(x)+\lambda(x)\right]
\end{aligned}
$$

$$
|\lambda(x)| \leqq 2\left|\frac{g^{(n)}(x)}{g^{(n-1)}(x)}\right| \times \sup _{\left[A_{3},+m !\right.}\left|F_{n+1}\right|
$$

août 1976. 


\section{Applications}

Exemple 1 : Prenons $g$ comme dans l'énoncé, $f: R \rightarrow C$ périodique centrée de période $T$. Posons $f_{(1)}=F, f_{(2)}=\Phi$; on sait que

$$
\|\Phi\|_{\infty} \leqq{ }_{4}^{T}\|F\|_{\infty} \text { (prop. 1). }
$$

Par suite,

$$
\int_{x}^{+\infty} f(t) g(t) d t=g(x)(-F(x)+\lambda(x))
$$

où

$$
|\lambda(x)| \leqq{ }_{2}^{T}\|F\|_{\infty}\left|\frac{g^{\prime}(x)}{g(x)}\right| .
$$

Exemple 2 : Soit $k \in R^{*}, \alpha>0$. Le corollaire 1 donne immédiatement

$$
\int_{x}^{+\infty} \frac{e^{i k t}}{t^{\alpha}} d t=-\frac{e^{i k x}}{i k} \frac{1}{x^{\alpha}}-\sum_{p=1}^{n} \frac{e^{i k x}}{(i k)^{p+1}} \frac{\alpha(\alpha+1) \ldots(\alpha+p-1)}{x^{\alpha+p}}+\frac{\lambda_{n}(x)}{x^{\alpha+n+1}},
$$

où

$$
\left|\lambda_{n}(x)\right| \leqq \frac{2 \alpha(\alpha+1) \ldots(\alpha+n)}{|k|^{n+2}}
$$

Notons $E$ la fonction «partie entière », et soit

Il vient

$$
\begin{gathered}
p=1+E(\alpha), \\
n(x)=E(|k| x) .
\end{gathered}
$$

$$
\left|\lambda_{n}(x)\right| \leqq \frac{2(n+p) !}{|k|^{n+1}|x|^{\alpha+n+1}} \text { pour tout } n \geqq 0 .
$$

En particulier,

$$
\int_{x}^{+\infty} \frac{e^{i k t}}{t^{\alpha}} d t=\sum_{0}^{n(x)} \frac{c_{n}}{x^{\alpha+n}} e^{i k x}+\rho(x),
$$

où

et

$$
c_{n}=\frac{-\alpha(\alpha+1) \ldots(\alpha+n-1)}{(i k)^{n+1}}
$$

$$
|\rho(x)| \leqq \frac{2(n(x)+p) !}{|k|^{n(x)+1}|x|^{n(x)+1}}
$$

Revue Française d'Automatique, Informatique et Recherche Opérationnelle 
dès que l'on a $x \geqq 1$. Donc

$$
\begin{aligned}
|\rho(x)| & \leqq \frac{2(n(x)+p)^{p-1}}{(|k| x)^{n(x)+1}} \sqrt{2 \Pi(n(x)+1)}\left(\frac{n(x)+1}{e}\right)^{n(x)+1}(1+\varepsilon(x)) \\
& \leqq C(1+|x|)^{p-(1 / 2)}\left(\frac{n(x)}{|k| x}\right)^{n(x)+1}\left(1+\frac{1}{n(x)}\right)^{n(x)+1} e^{-(n(x)+1)} \\
& \leqq C^{\prime}(1+|x|)^{p-(1 / 2)} e^{-|k| x} \leqq e^{-|k / 2| x}
\end{aligned}
$$

pour $x$ assez grand. Par développement en série de Fourier, on pourrait obtenir un résultat analogue pour une fonction $f$ continue périodique remplaçant la fonction $e^{\imath k x}$. De plus, il est également possible d'itérer le procédé et d'obtenir des estimations asymptotiques des intégrales "itérées » :

$$
\int_{x}^{+\infty} \int_{x_{1}}^{+\infty} \int_{x_{2}}^{+\infty} \ldots \int_{x_{n}}^{+\infty} \frac{e^{i k t}}{t^{\alpha}} d t d x_{n} d x_{n-1} \ldots d x_{1}
$$

Exemple 3 : Évaluation approchée de l'intégrale

$$
\int_{-\infty}^{+\infty} \sin \left(2 \Pi \sqrt{a^{2}+b^{2}\left(t-t_{0}\right)^{2}}\right) \sin c\left(\frac{2 t}{T_{0}}\right) d t \quad\left(\sin c(x)=\frac{\sin x}{x}\right),
$$

dans un domaine où

$$
\sup \left\{\frac{\left|t_{0}\right|}{T_{0}}, \frac{a}{b}\right\} \leqq C<+\infty
$$

et dans les deux cas-limites

$$
|b| \ll \frac{1}{T_{0}} \quad \text { et } \quad|b| \gg \frac{1}{T_{0}}
$$

On cherche le développement asymptotique de

$$
\int_{x}^{+\infty} \frac{e^{ \pm i t} e^{ \pm i \varepsilon} \sqrt{a^{2}+t^{2}}}{t+t_{0}} d t \quad \text { pour } \quad x \rightarrow+\infty
$$

Pour ramener cette étude au théorème 1 , on peut écrire :

$$
\begin{aligned}
e^{ \pm i x} & e^{ \pm i \varepsilon} \sqrt{\overline{a^{2}+x^{2}}} \\
& =e^{i( \pm 1 \pm \varepsilon) x} e^{ \pm i \varepsilon\left(\sqrt{\left.a^{2}+x^{2}-x\right)}\right.} \\
& =e^{i( \pm 1 \pm \varepsilon) x}\left[1+\left[ \pm i \varepsilon\left(\sqrt{a^{2}+x^{2}}-x\right)\right]+\frac{i^{2}}{2 !}\left[\varepsilon\left(\sqrt{a^{2}+x^{2}}-x\right)\right]^{2}+\ldots\right]
\end{aligned}
$$

et à partir de là chercher une primitive d'ordre 2 bornée.

août 1976. 
D'une façon générale, on a le résultat suivant :

Proposimion 2 : Si $f$ est périodique de pulsation $\omega>0$, et si $\lambda$ a un développement limite d'ordre $n$ au voisinage de $0, f(x) \lambda(1 / x)$ a une primitive d'ordre $(n-1)$ bornée pour $x$ assez grand.

COROLlaIre 2:Si $\sum_{n \geqq 0} c_{n} \gamma_{n}$ est une série entière de rayon de convergence strictement positif, et si est différent de zéro, la fonction $e^{t \omega x} \sum_{n \geqq 0} c_{n} / x^{n}$, définie pour $x$ assez grand, a des primitives bornées à tous les ordres.

Pour avoir des développements d'ordre supérieur, le corollaire du Théorème 1 est en pratique d'application assez malaisée, la recherche des majorants des primitives successives comportant des difficultés de calcul non triviales. De plus l'exemple 2 donne l'idée d'une possibilité de développement beaucoup plus précis, si on prend vraiment les moyens de calculer. Cette étude, liée à une régularité de type "analytique ", nous amène à un deuxième Théorème.

\section{DÉVELOPPEMENTS « D'ORDRE INFINI »}

1. Théorème $2:$ Soit $g:] 0,+\infty\left[\rightarrow C\right.$ une fonction de classe $C^{\infty}$ telle que

$$
\begin{gathered}
\forall n \in N, \quad \forall t \in\left[B,+\infty\left[,\left|g^{(n)}(t)\right| \leqq A n^{p} M^{n} n ! t^{-n \varepsilon-\eta},\right.\right. \\
\text { avec } \inf \{\varepsilon, \eta\}>0 .
\end{gathered}
$$

Alors, si l'on pose

$$
n(x)=E\left(\frac{|k|}{M} x\right) \quad \text { pour } \quad x>0
$$

( $k$ fixé dans $R-\{0\})$, l'intégrale $\int_{x}^{+\infty} e^{i k t} g(t) d t$ est convergente pour $x$ assez grand, et

$$
\int_{x}^{+\infty} e^{i k t} g(t) d t=-\sum_{n=1}^{n(x)} \frac{e^{i k x}}{(i k)^{n}} g^{(n-1)}(x)+\lambda(x)
$$

où

$$
|\lambda(x)| \leqq e^{-|k| / 2 M x^{\varepsilon}} \quad \text { pour } x \text { assez grand }
$$

pour $x$ assez grand.

Démonstration: (i) L'hypothèse implique que $g^{(n)} \rightarrow 0$ à l'infini pour tout entier $n$. De plus, pour $n$ assez grand, $\left|g^{(n)}(t)\right| \leqq K(n) t^{-2}$, donc en intégrant un nombre suffisamment grand de fois par parties, on constate la convergence de l'intégrale. 
(ii) Intégrant toujours par parties, il est facile de voir que :

$$
\int_{x}^{+\infty} e^{i k t} g(t) d t=-\sum_{n=1}^{N} \frac{e^{i k x}}{(i k)^{n}} g^{(n-1)}(x)+\lambda_{N}(x)
$$

avec

$$
\left|\lambda_{N}(x)\right| \leqq A\left|\frac{k}{M}\right|^{-N} N^{p} N ! \frac{x^{-N \varepsilon-\eta+1}}{N \varepsilon+\eta-1} \text { dès que } N \varepsilon \geqq 1 .
$$

En particulier si $N \varepsilon \geqq 2$, cette inégalité donne

$$
\left|\lambda_{N}(x)\right| \leqq A\left|\frac{k}{M}\right|^{-N} N^{p} N ! x, \quad x^{-N \varepsilon} \text { pour } x \text { assez grand. }
$$

Alors, pour $N$ assez grand, $(1 / N) N ! \leqq(N / e)^{N}$, et donc

Et si on choisit

$$
\left|\lambda_{N}(x)\right| \leqq A\left(\left|\frac{k}{M}\right| x^{\varepsilon}\right)^{-N} N^{N} N^{p+1} e^{-N} x
$$

$$
N=n(x)=E\left(\left|\frac{k}{M}\right| x^{\varepsilon}\right), \quad \text { alors } \quad N^{N}\left(\left|\frac{k}{M}\right| x^{\varepsilon}\right)^{-N} \leqq 1,
$$

d'où l'on déduit

$$
\left|\lambda_{N}(x)\right| \leqq A N^{p+1} e^{-N} x \leqq e A\left(\left|\begin{array}{c}
k \\
M
\end{array}\right| x^{\varepsilon}\right)^{p+1} e^{-(|k| / 2 M) x^{\varepsilon}}
$$

ce qui pour $x$ assez grand est majoré par $e^{-(k \mid / 2 M) x^{\varepsilon}}$.

\section{Applications}

Notons que les fonctions $g$ qui vérifient une inégalité de type (1) forment une sous-algèbre de celle des fonctions $\left.C^{\infty}:\right] 0,+\infty[\rightarrow C$. Plus précisément, si

$$
\begin{aligned}
& \left|g_{1}^{(n)}(t)\right| \leqq A_{1} n^{p_{1}} M_{1}^{n} n ! t^{-n \varepsilon_{1}-\eta_{1}}, \quad \varepsilon_{1}, \eta_{1} \geqq 0, \\
& \left|g_{2}^{(n)}(t)\right| \leqq A_{2} n^{p_{2}} M_{2}^{n} n ! t^{-n \varepsilon_{2}-\eta_{2}}, \quad \varepsilon_{2}, \eta_{2} \geqq 0,
\end{aligned}
$$

pour $t \geqq B \geqq 1$, on a

$$
\mid\left(g_{1} g_{2}^{(n)}(t) \mid \leqq A n^{p} M^{n} n ! t^{-n \varepsilon-\eta}\right.
$$

avec

$$
\begin{gathered}
M=\max \left\{M_{1}, M_{2}\right\}, \quad \eta=\eta_{1}+\eta_{2}, \\
\varepsilon=\min \left\{\varepsilon_{1}, \varepsilon_{2}\right\}, \quad p=p_{1}+p_{2}+1, \quad A=A_{1} A_{2} .
\end{gathered}
$$

août 1976. 
Par exemple, si $\varphi$ est une fonction analytique dans un voisinage de 0 avec $\varphi(0)=0$, et $\beta>0, \varphi\left(t^{-\beta}\right)$ est une fonction de ce type. En effet, on a

$$
\begin{gathered}
g(t)=\varphi\left(t^{-\beta}\right)=\sum_{p>0} c_{p} t^{-p \beta} \\
\left|g^{(n)}(t)\right| \leqq \sum_{p>0} p \beta(p \beta+1) \ldots(p \beta+n-1)\left|c_{p}\right| t^{-p \beta-n} \\
=t^{-n-\beta} \sum_{p \geqq 1} p \beta(p \beta+1) \ldots(p \beta+n-1)\left|c_{p}\right| t^{-(p-1) \beta} .
\end{gathered}
$$

Soit $N=E(\beta)+1$. On remarque que

$$
\begin{aligned}
& p(p \beta+1) \ldots(p \beta+n-1) \\
& \quad \leqq p N(p N+1) \ldots(p N+n-1)=n ! C_{p N+n-1}^{n} \leqq 2^{p N+n-1} n !
\end{aligned}
$$

d'où

$$
\left|g^{(n)}(t)\right| \leqq t^{-n-\beta} n ! 2^{n-1+N} \sum_{p \geqq 1}\left|c_{p}\right|\left(\frac{2^{N}}{t^{\beta}}\right)^{p-1} .
$$

Or pour $t$ assez grand, l'expression

$$
\sum_{p \geqq 1}\left|c_{p}\right|\left(\begin{array}{c}
2^{N} \\
t^{\beta}
\end{array}\right)^{p-1}
$$

est bornée. Plus précisément, si on pose

$$
\Phi(X)=\sum_{p \geqq 1}\left|c_{p}\right| X^{p-1} .
$$

on a pour $t$ assez grand

$$
\left|g^{(n)}(t)\right| \leqq(1+\Phi(0)) 2^{N-1} 2^{n} n ! t^{-n-\beta} .
$$

Pour $0 \leqq \alpha<1$, posons $g_{1}(t)=e^{t t^{\alpha}}$. Alors, notant $\varepsilon=1-\alpha$, on obtient :

$$
\begin{aligned}
g_{1}^{(n)}(t)=\left\{\begin{array}{r}
n \sum_{p=0}^{n-1} K_{n}^{p}[(n-p) \varepsilon][(n-p) \varepsilon+1] \ldots \\
\left.[(n-p) \varepsilon+p-1] t^{-(n-p) \varepsilon-p}\right\} e^{i t^{\alpha}},
\end{array}\right.
\end{aligned}
$$

où les coefficients $K_{p}^{n}$ vérifidnt la relation de récurrence

$$
K_{n+1}^{p+1}=-K_{n}^{p}+i \alpha K_{n}^{p+1} \quad\left(\text { avec } K_{n}^{n}=0\right) .
$$

En particulier, $K_{n}^{n} \mid \leqq C(1+\alpha)^{n}$ indépendamment de $p$, et donc

$$
\left|g_{1}^{(n)}(t)\right| \leqq C n(1+\alpha)^{n} n ! t^{n \varepsilon} \quad \text { pour } t \geqq 1 .
$$

Par suite, si $g(t)=t^{-\beta} e^{-s t^{\alpha}}, \beta>0$. on a

$$
\left|g^{(n)}(t)\right| \leqq A n^{2} 2^{n} n ! t^{-n(1-\alpha)-\beta} .
$$

Revue Française d'Automatique, Informatique et Recherche Opérationnelle 
Le cas $\beta=1, \alpha=1 / 2$, par exemple permet l'étude de l'intégrale

$$
\int_{x}^{ \pm \infty} \frac{e^{i t} e^{i a \sqrt{ }|i|}}{t} d t
$$

$a$ réel quelconque, lorsque $x \rightarrow+\infty$ ou $x \rightarrow-\infty$. Bien sûr, l'exemple 1 permet une étude plus précise de l'intégrale

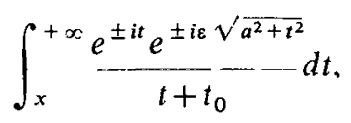

en écrivant

$$
\frac{e^{ \pm i t} e^{ \pm i \varepsilon \sqrt{a^{2}+t^{2}}}}{t+t_{0}}=e^{( \pm i \pm i \varepsilon) t} \times \frac{e^{ \pm i \varepsilon\left(\sqrt{a^{2}+t^{2}}-t\right)}}{t+t_{0}}
$$

et

$$
\sqrt{a^{2}+t^{2}}-t=t\left(\left(1+\frac{a^{2}}{t^{2}}\right)^{1 / 2}-1\right)=t\left[\left(1+\frac{a^{2}}{2 t_{2}^{2}}-\frac{a^{4}}{8 t^{4}}+\ldots\right)-1\right] .
$$

Par suite, l'expression

$$
\frac{1}{t+t_{0}} e^{ \pm \varepsilon \varepsilon \backslash^{\prime} \overline{\left.a^{2}+t^{2}-t\right)}}=\frac{1}{t}\left(1-\frac{t_{0}}{t}+\frac{t_{0}}{t^{2}}+\ldots\right) e^{ \pm i \varepsilon\left(a^{2} / 2 t-a^{4} / 8 t^{3} \ldots\right)}
$$

s'identifie pour $t>\sup \left\{, a, t_{0}\right\}$ à la somme d'une série du type étudié dans l'exemple 1 (avec $\beta=1)$. 